\title{
Pathophysiological Role of Trpv1 In Malignant Hyperthermia: Identification of New Variants
}

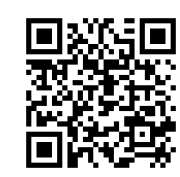

\author{
Nolwenn Tessier ${ }^{1 \#}$, Mallory Ducrozet ${ }^{1 \#}$, Sylvie Ducreux ${ }^{1}$, Julien Faure ${ }^{* 2}$ and Fabien Van Coppenolle*1 \\ ${ }^{1}$ CarMeN laboratory, France
}

${ }^{2}$ Laboratory of Genetic and Molecular Biochemistry, France

${ }^{\#}$ These authors contributed equally to this work

*These authors jointly supervised this work

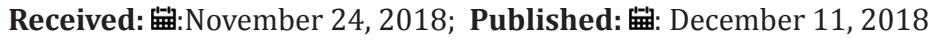

*Corresponding author: Fabien Van Coppenolle, France

Julien Faure, Laboratory of Genetic and Molecular Biochemistry, France

\begin{abstract}
Malignant hyperthermia is a pharmacogenetic disorder caused by volatile anesthetics that trigger severe muscle contraction and a hypermetabolic state. Outside triggered crisis, patients are asymptomatic. The molecular mechanisms of MH involve an uncontrolled increase of cytosolic $\mathrm{Ca}^{2+}$ concentration in skeletal muscles, which contributes to excessive muscle contraction and rigidity, increased body temperature, severe rhabdomyolysis and a generalized acidosis and hypermetabolic state. Mutations altering the function of the 2 main calcium channels involved in muscle contraction were so far linked to Malignant Hyperthermia. Recently, we showed that mutations in the Transient Receptor Potential Vanilloid 1 (TRPV1) cation channel could also be involved in MH. We propose that TRPV1, acting as a $\mathrm{Ca}^{2+}$ leak channel, is a target of volatile anesthetics such as isoflurane as well as a mechanism that could explain its implication in $\mathrm{MH}$.
\end{abstract}

Abbreviations: MH: Malignant Hyperthermia; TRPV1: Transient Receptor Potential Vanilloid 1; RyR1: Ryanodine Receptor type 1; Ca2+: Calcium

\section{Introduction}

Malignant Hyperthermia (MH) is a well described skeletal muscle disorder and a hypermetabolic response to inhalation of volatile anesthetics such as halothane, desflurane, sevoflurane and isoflurane. This disease is characterized by an increase of body temperature with cardinal associated clinical signs such as an elevation of expired $\mathrm{CO}_{2}$, increased ventilation rythm, muscle rigidity, rhabdomyolysis, acidosis, hyperkalemia and tachycardia, during anesthesia. Without appropriate care, $\mathrm{MH}$ crisis during anesthesia can be fatal. It is impossible to diagnose patient before surgery without exposing them to triggers, hence specific diagnostic testing was developed, based on the response to a muscle biopsy to volatile anesthetics, together with genetic testing of susceptible patients. It is difficult to estimate the prevalence of the mutations leading to $\mathrm{MH}$ but the incidence of $\mathrm{MH}$ episodes during anesthesia was reported between 1:10 000 and 1:250 000. Mutations in 3 genes were so far associated to $\mathrm{MH}$, involving 3 proteins of the calcium release complex leading to skeletal muscle contraction.

Most of the mutations are found in the RYR1 gene, that encodes the giant calcium channel of sarcoplasmic reticulum responsible for cytosolic Ca2+ raise in response to the cell depolarization [1,2]. More than 200 mutations in RyR1 were linked to MH, most of them being non-sense substitutions and conserved in three "hot spots" located in the N-terminal (C35-R614), central (D2129-R2458) and C-terminal regions (I3916-G4942), respectively. In accordance to the genetic bases of $\mathrm{MH}$, it was also described that patients with dominant or recessives forms of myopathies caused by RYR1 mutations (central core disease, multi-minicore disease, central nuclear myopathy and king-denborough syndrome) may also predisposed to episodes of MH [1] during anesthesia. However, although the genetic bases of $\mathrm{MH}$ are well studied, mutations are not found for 40 to $30 \%$ of the patients. Recently, we have were identified as new target gene for $\mathrm{MH}$, the ion channel TRPV1.

\section{Trpvi May Confer Susceptibility to Malignant Hyperther- mia}

TRPV1 (Transient Receptor Potential Vanilloid 1) is a noncationic channel that belongs to the ion channel family of the transient receptor potential. This channel is activated by heat (> $\left.42^{\circ} \mathrm{C}\right)$, acidosis $(\mathrm{pH}<6)$, lipids 3 and volatile anesthetics such as 
isoflurane. TRPV1 expression has been demonstrated in skeletal muscle and the channel is localized in the longitudinal part of the sarcoplasmic reticulum, where it plays the role of a reticular calcium leak channel $[3,4]$. Recently the residues in the outer pore region of TRPV1 channel that are critical for a specific activation by the volatile anesthetics like isoflurane [5] were precisely mapped (E600 and Y653). T, We then made the hypothesis that TRPV1 could be implicated in the triggering of malignant hyperthermia crisis and search for mutations in this gene in a cohort of MH patients. Two rare TRPV1 variants were identified in a cohort of $28 \mathrm{MH}$ patients. These patients had either suffer from a MH crisis or were classified as sensitive to $\mathrm{MH}$ on the base of a pre-symptomatic test relying on the contraction developed by a skeletal muscle biopsy upon either caffeine or halothane stimulation. Two rare genetic variations were found in two patients.

The first variant corresponds to a non-sense variation leading to the substitution of threonine 612 by a methionine (c.1834C>T; $p$. Thr612Met). The second one corresponds to the inframe deletion of three nucleotides leading to the deletion of aspargine 394 (c.1180-82delAAC;p.Asn394del). The impact of these two TRPV1 variations were first by ectopic expression in HEK-293 cells, and it was shown that their $\mathrm{Ca}^{2+}$ response to volatile anesthetic such as isoflurane cells was faster and more transient compared to a WT form of TRPV1.

To summarize, volatile molecules like isoflurane, halothane and sevoflurane are used in surgeries as anesthetics [1]. At cellular level, these molecules induce $\mathrm{Ca}^{2+}$ release through TRPV1 variant channels - given that their higher sensitivity to volatile anesthestics than wild type TRPV1 - from sarcoplasmic reticulum to cytoplasm [2]. $\mathrm{Ca}^{2+}$ released by TRPV1 channels directly activates RyR1mediated Ca2+ release [3]. This activation induces an uncontrolled increase in cytosolic $\mathrm{Ca}^{2+}[4]$, that allows an enhancement of skeletal muscle contraction [5]. This phenomenon leads to a muscle rigidity, hypermetabolism and causes a rise of temperature in muscle cells [6]. Finally, this increase in temperature would activate TRPV1 channels, triggering a higher $\mathrm{Ca}^{2+}$ leak from RS to cytosol [7]. Then, this leakage would activate RyR1-mediated $\mathrm{Ca}^{2+}$ release and would increase temperature in a vicious circle.

Heterologous expression of the human wild type TRPV1 and the two variants in skeletal muscle of trpv1-/- mice showed a longitudinal localization for the channels like that of the mouse wild type TRPV1. Furthermore, using $\mathrm{Ca}^{2+}$ imaging experiments and confocal microscopy, an enhanced reticular $\mathrm{Ca}^{2+}$ release was recorded during halogenated anesthetic perfusion, suggesting that mutated TRPV1 channels could trigger deleterious $\mathrm{Ca}^{2+}$ flux during anesthesia. In addition, when the TRPV1 antagonist capsazepine was used on a mouse model of MH (mice knock-in for the mutation Y524S in the RYR1 gene), it reduced significantly the heat-induce hypermetabolic response of the animal, showing that antagonizing TRPV1may affect the molecular mechanisms of an MH crisis linked to this RYR1 mutation.

\section{Conclusion}

It now established that TRPV1 is as a functional Ca2+ leak channel in adult skeletal muscle, sensitive to volatile anesthetics. The discovery in patients with MH of TRPV1 variants more sensitive to volatile anesthetics this channel strongly suggests that the TRPV1 channel plays a role in the molecular mechanism of the disease (Figure 1) and that it could be a new therapeutic target in triggered hyperthermia treatments.

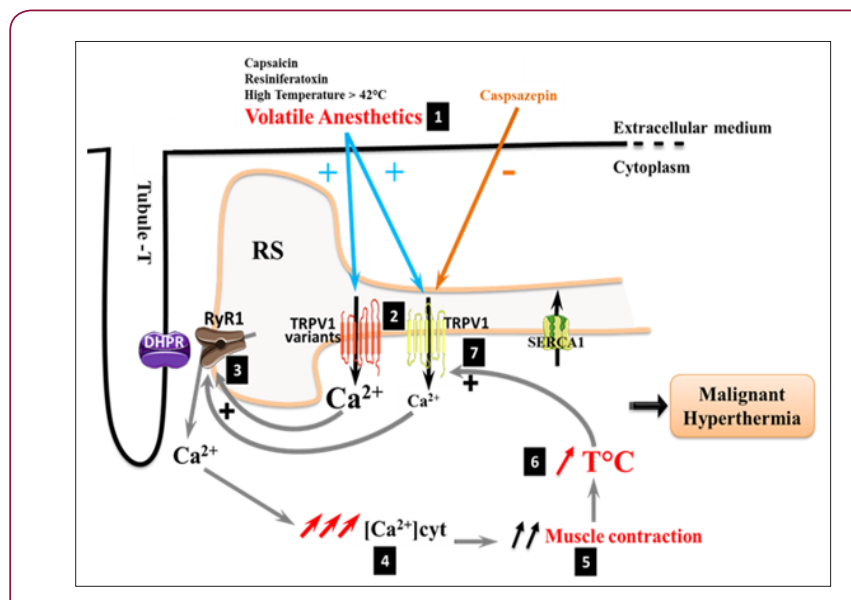

Figure 1: Proposed scheme of potential involvement of TRPV1 variants in malignant hyperthermia mechanism.

\section{Acknowledgment}

This study was supported by grants from the French Ministere of National Education and the National Institute of Health and Medical Research. The study was part of the Opera IHUB research program (ANR-10-IBHU-0004) within the "Investments of Future" operated by the French National Research Agency (ANR).

\section{References}

1. Rosenberg H, Pollock N, Schiemann A, Bulger T, Stowell K, et al. (2015) Malignant hyperthermia: A review. Orphanet J Rare Dis 10: 93.

2. Betzenhauser MJ, Marks AR (2010) Ryanodine receptor channelopathies. Pflugers Arch 460(2): 467-480.

3. Caterina MJ, Schumacher MA, Tominaga M, Rosen TA, Levine JD, et al. (1997) The capsaicin receptor: A heat-activated ion channel in the pain pathway. Nature 389(6653): 816-824.

4. Lotteau S, Ducreux S, Romestaing C, Legrand C, Van Coppenolle F, et al. (2013) Characterization of Functional TRPV1 Channels in the Sarcoplasmic Reticulum of Mouse Skeletal Muscle. PLoS ONE 8(3): e58673.

5. Kimball C, Luo J, Yin S, Hu H, Dhaka A, et al. (2015) The Pore Loop Domain of TRPV1 Is Required for Its Activation by the Volatile Anesthetics Chloroform and Isoflurane. Mol Pharmacol 88(1): 131-138.

6. FA Abeel, Sabine L, S Ducreux, Charlotte D, N Monnier, et al. (2018) TRPV1 variants impair intracellular $\mathrm{Ca}^{2+}$ signaling and may confer susceptibility to malignant hyperthermia. Genet Med Off J Am Coll Med Genet. 
ISSN: 2574-1241

DOI: $10.26717 / B J S T R .2018 .12 .002181$

Fabien Van Coppenolle, Julien F. Biomed J Sci \& Tech Res

(C) (P) This work is licensed under Creative

Submission Link: https://biomedres.us/submit-manuscript.php

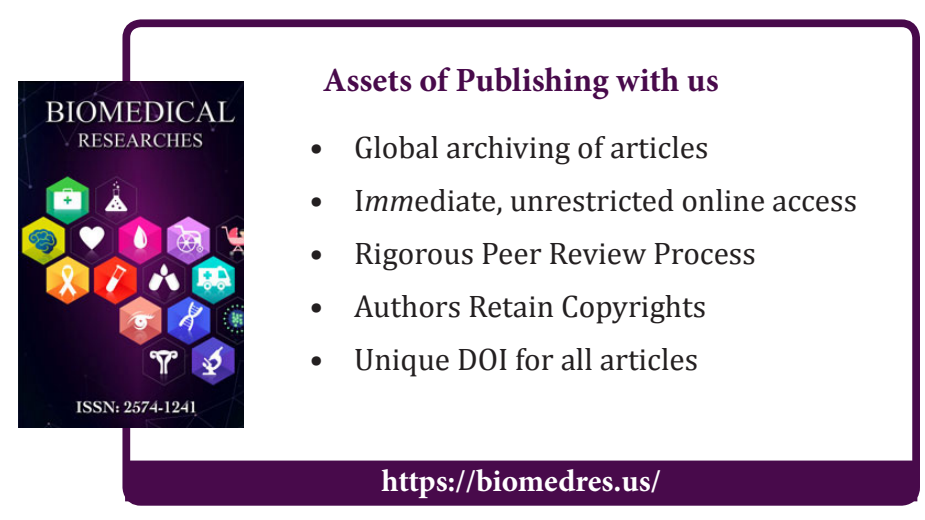

